\title{
An Einstein-like theory of gravity with a non-newtonian weak-field limit
}

\author{
M. Cadoni * \\ Università di Cagliari, Dipartimento di Fisica, \\ Cittadella Universitaria, 09042 Monserrato, Italy \\ and INFN, Sezione di Cagliari
}

\begin{abstract}
We propose a model describing Einstein gravity coupled to a scalar field with an exponential potential. We show that the weak-field limit of the model has static solutions given by a gravitational potential behaving for large distances as $\ln r$. The Newtonian term $G M / r$ appears only as subleading. Our model can be used to give a phenomenological explanation of the rotation curves of the galaxies without postulating the presence of dark matter. This can be achieved only by giving up at galactic scales Einstein equivalence principle.
\end{abstract}

*email: mariano.cadoni@ca.infn.it 
The weak-field limit of Einstein general relativity is Newton theory of gravity. In every textbook on general relativity one learns that for weak and static gravitational fields in the non relativistic approximation, the Einstein equations become the Poisson Equation for the Newtonian potential $\bar{\nabla}^{2} \phi=4 \pi G \rho$, which for a point-like source of mass $M$ has the solution $\phi=-M G / r$. Always in the same approximation, the geodesic equations of motion for a test particle in general relativity become Newton's second law.

The validity of the Newtonian limit of general relativity is unquestionable for distance scales ranging from the millimetre to solar system distances, where it is unambiguously supported by observations. For larger distances the situation is more involved. At galactic scales, the rotation curves of the galaxies cannot be explained by the Newtonian gravitational field generated by the visible matter (see for instance Ref. 1]). A Newtonian form of the potential is compatible with the observations only by postulating the existence of dark matter. Conversely, the observed rotation curves of the galaxies can be explained, without postulating the existence of dark matter, by modifying the Newtonian dynamics at small accelerations 2]. In particular, a non-newtonian gravitational potential behaving at galactic scales as $\phi \sim \ln r$ can explain the observational data.

More recently, radiometric data from the Pioneer, Galileo and Ulysses spacecrafts, have revealed anomalous accelerations, which could be explained by some modification of the Newtonian potential at small accelerations $\underline{3}$.

If one believes that the modification of Newtonian gravity at small acceleration is the right way to solve the puzzle of the rotation curves of the galaxies and the Pioneer anomaly, one has to find weak-field limits of general relativity (or some related theory of gravity) different from the Newtonian one. This turns out to be a very complicated task. It is easy to find Yukawa-like corrections to the Newtonian potential. This can be achieved for instance including higher-powers of the curvature tensor in the Einstein-Hilbert action [4. To our knowledge, logarithmic corrections to the Newtonian potential have been found only in the context of a bimetric theory of gravity [5]. Unfortunately, they appear only as a subleading term of an asymptotically linear gravitational potential.

In this paper we propose a model describing Einstein gravity coupled to a scalar field with an exponential potential. We show that in the weak-field limit our model admits static solutions given by a gravitational potential behaving for large distances as $\ln r$. The Newtonian term $-G M / r$ appears only as subleading. Our model can be used to give a phenomenological explanation of the rotation curves of the galaxies without postulating the presence of dark matter. Unfortunately, this can be achieved only by giving up at galactic scales a fundamental principle of the Einstein theory of gravity: the principle of equivalence.

We consider a system of two point-particles of mass $M$ and $m$, with $M>>m$ interacting with the gravitational field $g_{\mu \nu}$ and a scalar field $\varphi$. The gravitational interaction is described by the Einstein action. The scalar field has a potential $V(\varphi, \alpha)$, and its interaction with the point-particles is characterized by a coupling function $F(\varphi, \alpha)$. Notice that both the potential $V$ and the coupling function $F$ depend not only on $\varphi$ but also on some real parameter $\alpha$. Because $M$ is much bigger then $m$, the contribution of the mass $m$ as source of the 
gravitational field can be neglected. Thus, the only sources for the gravitational field are the mass $M$ and the scalar field $\varphi$. The mass $m$ will be considered as a test particle, whose motion is determined by the field configuration for $g_{\mu \nu}$ and $\varphi$.

The system is described by the Einstein-like action (we use units, where the speed of light $c=1$ and a signature $(-1,1,1,1)$ for the metric)

$$
\begin{aligned}
S & =\int d^{4} x \sqrt{-g}\left[\frac{1}{16 \pi G} R-\partial_{\nu} \varphi \partial^{\nu} \varphi+V(\varphi, \alpha)\right]+ \\
& -\sum_{a=1}^{2} m_{a} F_{a}(\varphi, \alpha) \int d t \sqrt{-g_{\mu \nu} \frac{d x_{a}^{\mu}}{d t} \frac{d x_{a}^{\nu}}{d t}}
\end{aligned}
$$

where $m_{1}=M, m_{2}=m$ and $x_{a}(t)$ are the positions of the two point-particles. The field equations describing the motion of the test particle with mass $m$ will be determined by taking variations of the action (1) with respect to $g_{\mu \nu}, \varphi$ and $x_{2}$. We get

$$
\begin{aligned}
& R_{\mu \nu}-\frac{1}{2} g_{\mu \nu} R=8 \pi G\left[T_{\mu \nu}^{(\varphi)}+(1+F) T_{\mu \nu}^{(M)}\right] \\
& 2 \nabla^{2} \varphi+\frac{\partial V}{\partial \varphi}=M \frac{\partial F}{\partial \varphi} \int d \tau \frac{\delta^{4}\left(x_{1}^{\nu}-x_{1}^{\nu}(\tau)\right.}{\sqrt{-g}} \\
& \frac{d^{2} x_{2}{ }^{\mu}}{d \tau^{2}}+\tilde{\Gamma}_{\rho \sigma}^{\mu} \frac{d x_{2}{ }^{\rho}}{d \tau} \frac{d x_{2}^{\sigma}}{d \tau}=0 .
\end{aligned}
$$

where $T_{\mu \nu}^{(\varphi)}, T_{\mu \nu}^{(M)}$ are the stress-energy tensors, respectively, for the scalar and for the source of mass $M$ and $\tilde{\Gamma}$ is a $\varphi$-dependent connection. Notice that in Eq. (2) we have neglected the contribution $T_{\mu \nu}^{(m)}$ of the test particle to the stress-energy tensor and to the equation for the scalar field and we have chosen $F_{2}=F_{1}-1=F . T_{\mu \nu}^{(\varphi)}$ and $T_{\mu \nu}^{(M)}$ are given by the following expressions

$$
\begin{aligned}
T_{\mu \nu}^{(\varphi)} & =2 \partial_{\mu} \varphi \partial_{\nu} \varphi-g_{\mu \nu}\left[(\partial \varphi)^{2}-V\right], \\
T_{\mu \nu}^{(M)} & =M \int d \tau u_{\mu} u_{\nu} \frac{\delta^{4}\left(x_{1}^{\alpha}-x_{1}^{\alpha}(\tau)\right)}{\sqrt{-g}},
\end{aligned}
$$

where $u_{\mu}$ is the quadrivelocity of the particle. $\tilde{\Gamma}$ is related to the usual affine connection $\Gamma$ by the relation,

$$
\tilde{\Gamma}_{\rho \sigma}^{\mu}=\Gamma_{\rho \sigma}^{\mu}+\frac{1}{2 F}\left(\partial_{\rho} F \delta_{\sigma}^{\mu}+\partial_{\sigma} F \delta_{\rho}^{\mu}-2 g_{\rho \sigma} g^{\mu \gamma} \partial_{\gamma} F\right)
$$

Let us now consider the usual weak-field, nonrelativistic, static limit of the field equations (2). Setting $g_{\mu \nu}=\eta_{\mu \nu}+h_{\mu \nu}$ with $h_{\mu \nu}<<1$, considering field configurations depending only on the spatial coordinates $x^{i}, i=1,2,3$, in the 
nonrelativistic limit, when the velocity of the particles $v<<1$ and $\left|T_{i j}\right|<<$ $\left|T_{00}\right|$, the field equations (2) give,

$$
\begin{aligned}
\bar{\nabla}^{2} \psi & =4 \pi G\left\{\left[(\bar{\nabla} \varphi)^{2}-V\right]+(1+F) \tilde{T}_{00}^{(M)}\right\} \\
\bar{\nabla}^{2} \varphi & =\frac{1}{2}\left(\frac{\partial F}{\partial \varphi} \tilde{T}_{00}^{(M)}-\frac{\partial V}{\partial \varphi}\right) \\
\frac{d^{2} \bar{x}}{d t^{2}} & =-\bar{\nabla} \psi-\frac{1}{F} \frac{\partial F}{\partial \varphi} \bar{\nabla} \varphi,
\end{aligned}
$$

where $\psi=-h_{00} / 2, \tilde{T}_{00}^{(M)}=M \delta^{3}\left(\bar{x}-\bar{x}_{1}\right)$, the bar indicates three-dimensional vectorial quantities, we have set $\bar{x}_{2}=\bar{x}$ and the differential operators are calculated with respect to the three-dimensional Euclidean metric.

The usual weak-field Newtonian limit can be trivially recovered setting in Eqs. (6) $F=0, V=0$ and picking the $\varphi=0$ solution for the scalar field equation. It is important to notice that there is an other way to recover the Newtonian limit from Eqs (6). Setting $F=0$ and choosing a potential $V$, which allows for solutions satisfying $(\bar{\nabla} \varphi)^{2}=V$, the scalar field decouples from the gravitational sector. The first and the third equations in (6) become, respectively, the Poisson Equation and Newton's second law. When the potential satisfies the equation $(\bar{\nabla} \varphi)^{2}=V$ consistency with the relation $\left|T_{i j}\right|<<\left|T_{00}\right|$, which determines the nonrelativistic limit, requires that the scalar field changes very slowly on the scale of distances we are considering. Using for instance spherical coordinates this means that the term $\left(\partial_{r} \varphi\right)^{2}$ in the $r r$ component of the stress-energy tensor can be neglected.

Let us now choose a potential and a coupling function with an exponential form

$$
V=\lambda^{2} \exp \left(-\frac{4 \sqrt{\pi}}{\alpha} \varphi\right), \quad F=\exp (2 \sqrt{\pi} \alpha G \varphi),
$$

where $\lambda^{2}$ is a constant with dimensions (mass)(length) ${ }^{-3}$. Because in the action (11) we define the potential $V$ with a sign opposite to the standard definition, our choice of Eq. (7) corresponds to a negative potential. A model of Einstein gravity coupled to scalar field with a negative exponential potential has been already proposed in the literature for solving the problem of the rotation curves of the galaxies 6, 7. The main difference between our model and that considered in Ref. [6]) is the fact that we introduce the coupling function $F(\varphi, \alpha)$.

Using Eqs. (7) and defining the new field

$$
\phi=\psi+2 \sqrt{\pi} G \alpha \varphi
$$

Eqs. (6) become

$$
\begin{aligned}
\bar{\nabla}^{2} \phi & =4 \pi G\left\{(\bar{\nabla} \varphi)^{2}+\left[1+\left(1+G \alpha^{2}\right) e^{2 \sqrt{\pi} G \alpha \varphi}\right] \tilde{T}_{00}^{(M)}\right\} \\
\bar{\nabla}^{2} \varphi & =2 \sqrt{\pi}\left(G \alpha e^{2 \sqrt{\pi} G \alpha \varphi} \tilde{T}_{00}^{(M)}+\frac{\lambda^{2}}{\alpha} e^{-\frac{4 \sqrt{\pi}}{\alpha} \varphi}\right) \\
\frac{d^{2} \bar{x}}{d t^{2}} & =-\bar{\nabla} \phi .
\end{aligned}
$$


From the third Equation in (9) it is evident that the field $\phi$ represents the potential that determines the force acting on the test particle. A spherical symmetric solution to the Eqs. (9) can be found placing the source-particle of mass $M$ at the origin of the coordinate system and using spherical coordinates $(r, \theta, \omega)$. The solution reads

$$
\begin{gathered}
\varphi=\frac{\alpha}{2 \sqrt{\pi}} \ln \left(\frac{2 \sqrt{\pi} \lambda}{\alpha} r\right), \\
\phi=G \alpha^{2} \ln C r-\frac{G M}{r},
\end{gathered}
$$

where $C$ is an arbitrary integration constant. The test particle will experience an acceleration

$$
a=-\frac{d \phi}{d r}=-\frac{G \alpha^{2}}{r}-\frac{G M}{r^{2}} .
$$

The potential $\phi$ of Eq. (11) has the $-G M / r$ Newtonian behavior only near $r=$ 0 . Its asymptotical, $r \rightarrow \infty$, behavior is logarithmic and therefore radically nonnewtonian. Far away from the source the Newtonian term is only subleading. The unpleasant feature of the gravitational potential (11) is its dependence from the parameter $\alpha$ parametrizing both the potential $V$ for the scalar field and the coupling function $F$. Our model can be phenomenologically relevant only if the logarithmic term appearing in Eq. (11) depends on the mass $M$ of the source. Moreover, to preserve the standard results of general relativity and its Newtonian limit at solar system scales, $\alpha$ should depend also on some threshold acceleration $a_{0}$, whose magnitude is such that the logarithmic term in Eq. (11) becomes relevant only at galactic scales. Formally, this can be achieved by writing the parameter $\alpha$ as function of $M$ and of the constants $G, \lambda: \alpha=\alpha(M, G, \lambda)$. If this is the case our model (11) can be used to solve the problem of the rotation curves of the galaxies without postulating the presence of dark matter.

The rotation curves at distance $r$ from the galactic core can be described by the equation

$$
v^{2}(r)=\frac{G M(r)}{r},
$$

where $v(r)$ is the velocity of a layer at distance $r$ and $M(r)$ is the total mass inside the layer. Observations are consistent with $M(r)$ behaving as

$$
M(r)=A r+B,
$$

where $A$ and $B$ are some constants (see for instance Ref.[1]). Using equations (12) and Eq. (13) one easily derives

$$
M(r)=\alpha^{2} r+M,
$$

in accordance with the experimental curve (14).

Fixing appropriately the form of the constant $\alpha$ our model can be used to derive the modified Newtonian dynamics (MOND) of Milgrom [2] as the 
weak-field limit of the Einstein-like model (1). MOND introduces a constant acceleration $a_{0} \sim 10^{-29} \mathrm{~cm}^{-1}$, such that the standard Newtonian dynamics is a good approximation only for accelerations $a>>a_{0}$. For $a \sim a_{0}$ MOND predicts that a test particle at distance $r$ from a mass $M$ experiences an acceleration [8]

$$
|a|=\frac{\sqrt{M a_{0} G}}{r} .
$$

This expression can be derived from our model setting

$$
\alpha^{2}=\sqrt{\frac{M a_{0}}{G}} .
$$

Using this equation we can see that the leading term in Eq. (12) reproduces exactly the MOND result (16). Simple dimensional analysis allows us to identify $a_{0}$ in terms of the two dimensional parameters $G, \lambda$ appearing in our model : $a_{0}=\lambda \sqrt{G}$.

For $a>>a_{0}$ we recover the standard Newtonian dynamics. In fact, $a>>a_{0}$ implies $\sqrt{G M} / r>>\sqrt{a_{0}}$. It follows that in this limit in Eq. (12) the Newtonian term $G M / r^{2}$ dominates with respect to the first term. Eq. (10) tells us that for $a>>a_{0}, \varphi \rightarrow-\infty$, which in turn implies $F=0$. Because the solution (10) satisfies $(\bar{\nabla} \varphi)^{2}=V$, the scalar field decouples in the weak-field limit from the gravitational sector and we obtain the standard Newtonian limit (see discussion after Eq. (6) ).

The Einstein theory of gravity with a negative cosmological constant can be obtained as a particular case of our model. Taking in Eqs. (17) the limit $\alpha \rightarrow-\infty$ we have $F=0$ and $V=\lambda^{2}$. Picking the $\varphi=0$ solution of the field equation for the scalar, the action (11) becomes the Einstein-Hilbert action with a cosmological constant.

It is obvious that identifying the parameter $\alpha$ in terms of the mass of the source we are giving up at galactic scales a fundamental principle of the Einstein theory of gravity: the principle of equivalence. This is not immediately evident in our simplified model (1) because we are considering the motion of a test particle in the gravitational field generated by the source of mass $M$. The source is completely characterized by its gravitational mass whereas for the test particle only its inertial mass can be relevant. Because the equations of motion of the test particle (2) turn out to be independent of its mass, one could be erroneously led to conclude that the equivalence principle still holds. This is not true. The breakdown of the equivalence principle will immediately show up when we try to describe in a self consistent way the mutual interaction of the two masses.

Apart from the breakdown of the equivalence principle, a gravitational theory described by the action (1) in which the parameter $\alpha$ is a function of the mass $M$ of the source poses also huge interpretation problems. Implicitly we are assuming the existence of a "cosmic" scalar field $\varphi$ whose self-coupling (the potential $V$ ) and its coupling with the matter (the coupling function $F$ ) are determined by the distributions of the sources for the gravitational field. We do 
not have a definite prescription of how the information about the distribution of matter has to be encoded on the form of the functions $F$ and $V$. There is no general argument, no principle, behind our Eq. (17). Its only justification is the accordance with the observed rotation curves of the galaxies. For this reason our model, at least in the present context, cannot have a fundamental but just a phenomenological character. Independently of the fundamental, still unknown physics that could lie behind our phenomenological model, it is likely that the information about the distribution of matter has to be encoded in the cosmic field, trough the form of the functions $V(\varphi, M)$ and $F(\varphi, M)$, in a non local way. We are leaving the Einsteinian paradigm and moving toward a Machian description of the gravitational interaction.

Our model (11) can be considered as a particular case of a scalar-tensor theory of gravity. It is well known that scalar-tensor theories of gravity can reproduce the standard phenomenology of general relativity at solar-system scales (perihelion shift of Mercury and bending of light by the sun), only for particular values of the parameters entering in the theory [9. It is therefore necessary to check that our model (1) with potential and coupling function given by Eq. (7), apart from explaining the rotational curves of the galaxies, can also get through the standard tests of general relativity at solar-system scale. This point will be discussed in a forthcoming publication.

We conclude by noticing the striking similarities of our model with the "quintessence" models proposed in cosmology for solving the dark energy problem [10, 11. This problem can be solved by introducing in the Einstein theory a scalar field (the quintessence field) with an exponential potential. The main difference with our model, apart from the presence of the coupling function $F$, is the sign of the potential, which using the standard notation is positive for quintessence models and negative for our model. It is amusing that both dark matter and dark energy problem can be solved, at least phenomenologically, by introducing in the Einstein action scalar fields with exponential potentials.

\section{Acknowledgments}

We thank S. Mignemi, M. Cavagliá, M. Lissia and D. L. Wiltshire for discussions and valuable comments.

\section{References}

[1] V. Trimble, Ann. Rev. Astron. Astrophys. 25 (1987) 425.

[2] M. Milgrom, Astrophys. J. 270 (1983) 365.

[3] J. D. Anderson, P. A. Laing, E. L. Lau, A. S. Liu, M. M. Nieto and S. G. Turyshev, Phys. Rev. Lett. 81 (1998) 2858 arXiv:gr-qc/9808081.

[4] K. S. Stelle, Gen. Rel. Grav. 9 (1978) 353.

[5] O. Bertolami and J. Paramos, arXiv:gr-qc/0310101. 
[6] T. Matos, F. S. Guzman and D. Nunez, Phys. Rev. D 62 (2000) 061301 arXiv:astro-ph/0003398.

[7] T. Matos and F. S. Guzman, Class. Quant. Grav. 18 (2001) 5055 arXiv:gr-qc/0108027.

[8] M. Milgrom, Annals Phys. 229 (1994) 384 arXiv:astro-ph/9303012.

[9] R. V. Wagoner, Phys. Rev. D 1 (1970) 3209.

[10] R. R. Caldwell, R. Dave and P. J. Steinhardt, Phys. Rev. Lett. 80 (1998) 1582 arXiv:astro-ph/9708069.

[11] T. Barreiro, E. J. Copeland and N. J. Nunes, Phys. Rev. D 61 (2000) 127301 arXiv:astro-ph/9910214. 